Table 1 (Brainerd). Memory advantages that make intuitive reasoning advanced

\begin{tabular}{|c|c|}
\hline Advantange & Description \\
\hline Memory availability & $\begin{array}{l}\text { Intuitive reasoning processes the types } \\
\text { of memories (gist) that are stable } \\
\text { over time. The types of memories } \\
\text { processed by logical reasoning are } \\
\text { not stable. }\end{array}$ \\
\hline Memory accessibility & $\begin{array}{l}\text { The gist memories that intuition } \\
\text { processes are accessible by a broad } \\
\text { range of retrieval cues. }\end{array}$ \\
\hline Memory malleability & $\begin{array}{l}\text { The sketchy nature of gist memories } \\
\text { makes them especially easy to } \\
\text { transform into solutions during } \\
\text { reasoning. }\end{array}$ \\
\hline Processing simplicity & $\begin{array}{l}\text { The simplicity of gist memories makes } \\
\text { intuitive reasoning relatively } \\
\text { uncomplicated. }\end{array}$ \\
\hline Processing accuracy & $\begin{array}{l}\text { Intuitive processing of gist memories is } \\
\text { usually just as accurate (and is often } \\
\text { more accurate) than logical } \\
\text { processing of more elaborate, } \\
\text { detailed memories. }\end{array}$ \\
\hline Processing effort & $\begin{array}{l}\text { Intuitive processing of gist memories is } \\
\text { easier to execute than logical } \\
\text { processing of more elaborate, } \\
\text { detailed memories. }\end{array}$ \\
\hline
\end{tabular}

Concerning assumption (b), FTT explains reasoning illusions by isolating structural properties of reasoning problems that interfere with three general stages of cognitive processing: (1) storing the correct problem representation (its "gist"), (2) retrieving that representation and the appropriate processing operations on reasoning problems, and (3) executing the steps that are required for the processing operations to deliver solutions. This approach is exemplified in FTT's explanations of many types of reasoning failures, such as arithmetic errors (Brainerd \& Reyna 1988) and transitive inference errors (Reyna \& Brainerd 1990).

Concerning assumption (c), extensive research was conducted on the cognitive mechanisms that cause errors in the family of illusions to which base-rate neglect belongs: inclusion illusions. Much of that work involved a prototypical task that produces such errors: Piaget's class-inclusion problem. The mechanisms that were identified were then generalized to base-rate problems, conjunction problems, probability problems, expected-value problems, and other tasks in the inclusion illusions family. Classinclusion problems are structurally simple but cognitively impenetrable: Children are presented with an array of objects, subdivided into two (or more) familiar sets, such as 7 cows and 3 horses, that belong to a common superordinate set (10 animals), and are asked "Are there more animals or more cows?" Young children consistently respond: "more cows." This error persists for many years, with the error rate at age 10 still being 50\% (Winer 1980), and adults routinely make the error on slightly more complex versions of the problem (Reyna 1991). Why are such problems so difficult?

The answer that emerged, following many experiments (e.g., Brainerd \& Reyna 1990; 1995), is that nested sets interfere massively with the aforementioned processing stages. Reyna (1991) summarized the cognitive effects as follows: "processing focuses on the subset mentioned in the question, the superordinate set recedes, and the question appears to involve nothing more than ... a subset-subset comparison" (p. 325). These effects were found to be rooted in the fact that problems in the inclusion illusions family have two-dimensional structures, with one dimension (the subset-subset) being salient and easy to process and the other (the subset-superordinate set), which is crucial to solution, being obscure. The obscurity is caused by the containment relation, which creates "mental booking" problems in which subsets disappear whenever the mind focuses on the superordinate set and the superordinate set disappears whenever the mind focuses on the subsets. Yet, correct reasoning demands subset-superordinate set comparisons. Reyna went on to formulate the denominator neglect model, wherein this difficulty was posited as the cause of base-rate neglect, the conjunction fallacy, and other errors that arise from comparing numerical parts to numerical wholes. The term "denominator" referred to the fact that denominator information is ignored because denominators are obscure wholes of part-whole relations.

A last point that illustrates the deep parallels between the Reyna and the Barbey-Sloman models is the centrality of formatting manipulations in tests of the models. These are manipulations that make problem structure more transparent and, crucially, enhance the salience of subset-superordinate set relations. Reyna noted that her model predicts that such manipulations reduce the mental bookkeeping problem and, therefore, should significantly reduce errors. A formatting manipulation called tagging provided dramatic confirmation. Young children, who failed problems such as the animal example across the board, performed nearly perfectly when simple tags (e.g., a hat on each animal's head, a bow on each animal's tail) were affixed to all the members of each subset, so that the superordinate set was just as salient as the subsets. Likewise, B\&S stress the importance of presentation formats that allow "accurate representation in terms of nested sets of individuals" (target article, Abstract) in baserate problems. With respect to the most effective presentation formats that they discuss, these formats, too, are ones that ought to reduce the mental bookkeeping problem of nested sets.

Although the Barbey-Sloman model is anticipated to a remarkable degree by the Reyna model, the dual-process frameworks that lie behind the models are different. The Barbey-Sloman framework is the traditional System 1/System 2 approach, which treats intuition as a primitive form of thinking that cognitive development and expertise evolve away from. The Reyna framework is FTT, which treats intuition as an advanced mode of thinking that cognitive development and expertise evolve towards. That intuition is advanced by virtue of memory considerations, though different ones than those which figure in the BarbeySloman model (see Table 1).

\section{Omissions, conflations, and false dichotomies: Conceptual and empirical problems with the Barbey \& Sloman account}

\section{DOI: $10.1017 / S 0140525 X 07001690$}

\section{Gary L. Brase}

Department of Psychology, Kansas State University, Manhattan, KS 66506-5302.

gbrase@ksu.edu

Abstract: Both the theoretical frameworks that organize the first part of Barbey \& Sloman's (B\&S's) target article and the empirical evidence marshaled in the second part are marked by distinctions that should not exist (i.e., false dichotomies), conflations where distinctions should be made, and selective omissions of empirical results - within the very studies discussed - that create illusions of theoretical and empirical favor.

Theoretical frameworks. The number of contrasting theoretical frameworks that Barbey \& Sloman $(B \& S)$ face is impressive on the face of it - four against one.But are all these distinctions appropriate or scientifically useful? The short 
way to demonstrate they are not is to simply note that the same theorists (Gigerenzer, Cosmides, and Tooby) are repeatedly invoked for all of the first three frameworks.

The longer demonstration is to dismantle these accounts sequentially. There is the "Mind as Swiss army knife" framework that is distinguished by unavailability to conscious awareness or deliberate control (cognitive impenetrability), and then there is the "Natural frequency algorithm" framework that is informationally encapsulated. But wait; those distinguishing characteristics are actually imposed by others (e.g., Fodor 1983) and rejected by the actual theorists under consideration here (e.g., Cosmides \& Tooby 2003; Duchaine et al. 2001; Ermer et al. 2007; Tooby et al. 2005; Tooby \& Cosmides 2005). The first and second theoretical frameworks therefore collapse into a third one.

The third framework (incorporating the previous two), is a "Natural frequency heuristic" account, and is probably closest to the one actual and appropriate opposing view for $B \& S$. The fourth framework ("Non-evolutionary natural frequency heuristic") suggests that an appropriate position is to willfully disregard all evolutionary factors that have influenced the structure and function of the human mind. One can question the nature of the cognitive structures generated by evolutionary selection pressures, but it is not scientifically legitimate to simply deny evolution and replace viable evolutionary explanations with "one way or another, people can appreciate and use [natural sampling]" and that somehow "gives rise to" Bayesian reasoning (sect. 1.2.4). Such vague descriptive explanations would have effectively stagnated our understanding of visual processing or language acquisition, and will have that effect on other cognitive phenomena if unchecked.

This leaves us with two real frameworks, the final "nested sets/ dual processes" framework and an ecological rationality framework - the two frameworks of the target article's title. It is not so much that there is no possibility of other frameworks, but rather, that the ones described by B\&S are not useful.

The empirical literature. Having constructed artificial required properties for the theoretical frameworks of others, B\&S then tout the inability of those shackled frameworks to account for empirical results. As easy as this should be, given such a set up, it is nevertheless seriously flawed. Due to space constraints, I focus here on how my own research is considered within this target article. B\&S use the findings of Brase et al. (2006) to support a claim that "Bayesian inference depends on domain general cognitive processes" that are strategically employed (sect. 2.1). This was not the original purpose, findings, or conclusions of our work - and for good reason. As B\&S note in that very same section, there have been differences in absolute performance levels on Bayesian reasoning tasks, when comparing across research programs. These different research programs, however, had used different participants and different methods for obtaining those participants (e.g., paid versus classroom activity participation). Brase et al. (2006) sought to determine the effects of participant selection and recruitment methods on performance on such tasks, and found that there were, indeed, significant effects that were capable of accounting for all the differences in previous works. In summary, B\&S make a confusion between performance and competence (Chomsky 1965) when they try to infer cognitive abilities and structures from data showing that incentives affect performance (see also Crespi 1942; 1944).

There also appears to be some confusion about the nature of natural sampling and natural frequencies (i.e., naturally sampled frequencies). The use of a consistent reference class (sect. 2.3), also called using a partitive structure, nested sets, or subset relations, are all linguistic twists on what is, in fact, natural sampling (a point made many times by myself and others; Brase 2002a; 2002b; Brase \& Barbey 2006; Gigerenzer \& Hoffrage 1999; Hoffrage et al. 2002). Natural sampling refers to the sequential acquisition of information (as in a natural environment) along with categorization of that information into meaningful, often overlapping, groups (see Brase et al. 1998 for some limitations on easily constructible categories.).

This confusion is starkly illustrated when B\&S try to re-define the numerical formats used in Brase (2002b). First, natural frequencies are equated with simple frequencies by providing an incorrect example of the former (this example belongs to $B \& S$ and is not, as they claim, an inconsistency with the literature on the part of Brase 2002b). In direct contradiction to $B \& S$, a single numerical statement such as the simple frequencies used in Brase (2002b) cannot be identified as having a natural sampling structure. Second, B\&S point out - correctly - that percentages can express single-event probabilities, but they then carry this too far in concluding that this is the only thing that probabilities can express. Indeed, as pointed out in Brase (2002b), percentages are also referred to as "relative frequencies" because they can be understood as frequencies that are normalized to a reference class of 100 (e.g., as when one says " $90 \%$ of my students understand this topic").

With B\&S having misconstrued natural frequencies into simple frequencies, and misconstrued relative frequencies into probabilities, it is almost possible to claim that the results of Brase $(2002 \mathrm{~b})$ indicate that single event probabilities are perceived equally well compared to natural frequencies. The remaining necessary manipulation is for $\mathrm{B} \& \mathrm{~S}$ to also completely omit the other numerical format conditions used in Brase (2002b), which included actual single-event probabilities (and, no, these actual single-event probabilities were not understood as well or clearly as simple frequencies and relative frequencies).

\section{Why frequencies are natural}

\section{DOI: $10.1017 /$ S0140525X07001707}

\section{Brian Butterworth \\ Institute of Cognitive Neuroscience, University College London, London WC1N $3 A R$, United Kingdom. \\ b.butterworth@ucl.ac.uk}

Abstract: Research in mathematical cognition has shown that rates, and other interpretations of $\mathrm{x} / \mathrm{y}$, are hard to learn and understand. On the other hand, there is extensive evidence that the brain is endowed with a specialized mechanism for representing and manipulating the numerosities of sets - that is, frequencies. Hence, base-rates are neglected precisely because they are rates, whereas frequencies are indeed natural.

Barbey \& Sloman $(B \& S)$ are to be congratulated for laying out the explanations for base-rate neglect so clearly and systematically. However, to a researcher not from the field of normative rationality research, but from the field of mathematical cognition, it is surprising that none of the explanations make reference to what is known about how we process numerical quantities (Butterworth 2001). From this perspective, another type of explanation can be proposed for base-rate neglect. It is in the word "rate." Rates can be expressed formally as $\mathrm{x} / \mathrm{y}$, and it is well known from research in mathematical cognition and education that humans are very bad at understanding $\mathrm{x} / \mathrm{y}$ however it is interpreted - as a fraction, as a proportion, or as a rate. For example, it is well known that children find it hard to learn and understand fractions and simple operations on them (Bright et al. 1988; Hartnett \& Gelman 1998; Mack 1995; Smith et al. 2005). It has also been found that most third and fourth graders cannot order fractions by size and cannot explain why there are two numbers in a given fraction (Smith et al. 2005). In particular, they seem to have trouble getting away from whole numbers - for example, when they say that $1 / 56$ is smaller than $1 / 75$ because 56 is smaller than 75 